\title{
Zur pragmatischen Fundierung semantischer Strukturen am Beispiel der Dialoglogik
}

Das gegenwärtige Interesse an pragmatischen Eigenschaften sprachlicher Ausdrücke - ihrer kommunikativen Funktion - hat in Verbindung mit dem Versuch, Abgrenzungskriterien gegenüber ihren semantischen Eigenschaften - ihrer repräsentativen Funktion - anzugeben, dazu geführt, zwei miteinander konkurrierende Forschungsstrategien zu verfolgen:

(a) den Frege-Weg einer Semantisierung der Pragmatik und

(b) den Wittgenstein-Weg einer Pragmatisierung der Semantik.

Im ersten Falle werden die sprachlichen Ausdrücke mit speziellen Ausdrücken für die Umstände ihrer Äußerung, insbes. Zeit und Ort, indiziert. Dazu müssen die semantischen Relationen, insbes. Referenz und Sinn, also Benennen und Unterscheiden, bereits zur Verfügung stehen, was durch Rückgriff auf die moderne Logik geschieht. Es wird nämlich die Fregesche Satzanalyse benützt, die darauf beruht, den mathematischen Argument-Funktionszusammenhang so auf - zunächst elementare Sätze anzuwenden, daß ein Prädikatsausdruck zum Ausdruck einer Aussagefunktion mit den Subjektausdrücken als ihren Argumenten bzw. Argumentausdrücken erklärt wird, was dazu führt, neben den logischen Partikeln grundsätzlich nur Nominatoren (singular terms) und Prädikatoren (general,terms) zu berücksichtigen. In der generativen Semantik ist daraufhin die in der generativen Transformationsgrammatik angenommene Tiefenstruktur eines Satzes konsequenterweise mit dessen logischer Struktur identifiziert worden.

Im zweiten Fall werden die Bedeutungsbeziehungen aus Sprachhandlungen im Kontext der übrigen Handlungen, also den möglichen kommunikativen Funktionen menschlicher Handlungszusammenhänge in bis heute noch nicht hinreichend analysierten Schritten gewonnen. Bei Wittgenstein wird dafür in den "Philosophischen Untersuchungen" das Konzept der Sprachspiele entwickelt, das bislang bei den Sprechakttheoretikern nur zögernde Aufnahme gefunden hat, vermutlich deshalb, weil, insbes. bei J.L. Austin und J.R. Searle, die klassische logische Analyse, die im propositionalen Gehalt einer Außerung gesucht wird und sich eines Frege'schen Aufbaus bedient, als Grundlage nicht preisgegeben werden soll, Semantik um Pragmatik nur ergänzt wird. 
Ich vertrete hier den Wittgenstein-Weg (eine zusammenfassende Darstellung in meinem Essay "Sprachphilosophie", in: Lexikon der Germanistischen Linguistik, hrsg. H.P. Althaus u.a., Tübingen 21980 ), wobei die Dialoglogik ein Testfall war, nämlich im harten Kern der Semantik, bei der synsemantischen Behandlung der logischen Partikeln, die Pragmatisierung als sinnvoll, also verständnisfördernd vorzuführen. Das logische Schließen selbst dann als eine praktische Anwendung logisch-semantischer Strukturen aufzufassen, war nie zweifelhaft, eher ließe sich fragen, ob nicht eben diese der Praxis vorausliegenden semantischen Strukturen wie sie in den Wahrheitswerttabellen für die logischen Partikeln üblicherweise notiert sind, ihrerseits erst aus einer Argumentationspraxis zu gewinnen sind. Genau so ist die Dialoglogik weithin - und die Terminologie ihrer Verfasser hat dieses Verständnis noch befördert (vgl. die Zusammenfassung der bisherigen Entwicklung und ihrer Resultate in: P.Lorenzen/K. Lorenz, Dialogische Logik, Darmstadt 1968) - verstanden worden (zuletzt in C.F. Gethmanns "Protologik. Untersuchungen zur formalen Pragmatik von Begründungsdiskursen", Frankfurt 1979): sie galt und gilt als ein Versuch, mit den in ihr verwendeten Spielregeln eine Argumentationspraxis zu explizieren, der es erlaubt, die sonst grundsätzlich als undefiniert zu belassenden Begriffe inhaltlicher wie auch logischer Wahrheit explizit und präzise zu definieren. Mit dieser Unterstellung, die Dialogspielregeln explizierten das - vorsystematische - Argumentieren oder sollten es doch wenigstens tun, ist die Frage nach einer pragmatischen Fundierung semantischer Strukturen, hier: der logischen Partikeln, auf die These zugeschnitten worden, daß eine pragmatische Rechtfertigung oder) Begründung (der Logik, insbesondere eine pragmatische Rechrfertigung oder > Begründung 〈 der bislang nicht hinreichend diskutierten Spielregeln in der dialogischen Fassung der Logik, damit gleichwertig sei, in der Theorie des logischen Schließens Argumentationsprobleme (zunächst) des Alltags und (dann) auch der Wissenschaft zu lösen. Solange nun der in dieser These ausgedrückte Zusammenhang selbst aber noch nicht hinreichend rekonstruiert sei, können die Begründungen für die Dialogspielregeln notgedrungen den Bereich bloß interner, an scheinbar allein formalen Problemen - z.B. spieltheoretischer Darstellbarkeit - orientierten und deshalb zurecht willkürlich erscheinenden Überlegungen nicht verlassen. Der Kern dieses Einwands, wie am deutlichsten in der "Protologik" von Gethmann herausgearbeitet worden ist, kann als Syntaxverdacht gegenüber der von der Dialoglogik beanspruchten Pragmatisierung der Semantik (am Beispiel der logischen Partikeln) gedeutet werden. Die spieltheoretische Behandlung der Logik wäre ähnlich der am Anfang der modernen Logik stehenden kalkültheoretischen Behandlung ein syntaktischer Zugriff zu semantischen Problemen, insbes. zum Wahrheitsbegriff, und nicht, wie beansprucht, 
ein Paradebeispiel für eine gelungene pragmatische Behandlung semantischer Probleme. Kein Wunder daher, so fährt die Kritik fort, daß der performative Aspekt einer mit dem Ziehen gemäß den Spielregeln aktualisierten Sprechhandlung undeutlich und in seiner für die gesuchte pragmatische Rechtfertigung der fraglichen Regel entscheidenden Rolle unaufgedeckt geblieben ist.

So richtig es nun ist, den der Spieltheorie entlehnten begrifflichen Rahmen für den dialogischen Aufbau der Logik nicht unbesehen als einen zugleich auch argumentationstheoretisch ausgewiesenen Rahmen hinzunehmen, so voreilig ist es, die Dialogführung selber, also eine Partie um eine Aussage nach den Dialogspielregeln, mit Begründungen oder auch nur Begründungsversuchen für diese Aussage zu identifizieren. Es war ja gerade der entscheidende Kunstgriff des dialogischen Ansatzes in der Logik, ein Aussagen charakterisierendes entscheidbares Prädikat zur Verfügung zu stellen, das an die Stelle der nicht generell - für alle Aussagebereiche - als entscheidbare Prädikate einführbaren 'wahr' bzw. 'falsch' treten kann: Aussagen sollen wenigsten dialogdefinit sein, wenn sie schon nicht als wertdefinit (zweiwertig) oder auch nur beweisdefinit (verifizierbar) bzw. widerlegungsdefinit (falsifizierbar) charakterisierbar sind. Diese Forderung ist mit der im spieltheoretischen Aufbau möglich gewordenen strikten Trennung von partiebezogenen und strategiebezogenen Überlegungen durchgesetzt worden: Gewinn und Verlust sind relativ zu einer beendeten Dialogführung - entscheidbare Prädikate über der Anfangsbehauptung, Wahrheit und Falschheit hingegen - als Gewinnenkönnen bzw. Verlierenmüssen unabhängig von der Dialogführung des Partners strategiebezogen und nicht mehr partiebezogen erklärt - im allgemeinen Fall natürlich nicht. Als Folge dieser Trennung zwischen Partieebene und Strategieebene wird es weiterhin möglich, eine begriffliche Differenzierung zwischen Sinnbestimmung und Geltungssicherung für Aussagen vorzunehmen, die traditionell ausgeschlossen war, weil der Sinn einer Aussage, d.i. der von ihr dargestellte Sachverhalt, grundsätzlich nicht anders zu erklären war als durch die Angabe der Wahrheitsbedingungen für diese Aussage. Das pragmatische Fundament für die Sinnbestimmung einer Aussage, durch die möglichen Argumentationen i n einer Dialogführung um diese Aussage präziert (Partieebene!) ist damit deutlich vom pragmatischen Fundament für die Geltungssicherung einer Aussage, durch die Argumentationen ü b e r die Dialogführungen mit dem Ziel, die Existenz einer Gewinnstrategie nachzuweisen, charakterisiert (Strategieebene!), unterschieden. Im ersten Fall geht es um Semantik, die natürlich als pragmatisch fundiert behandelt wird, während nur im zweiten Fall es ausdrücklich auch um Pragmatik im herkömmlichen Sinne geht. Allerdings muß man dann die vieldiskutierte 
Zweideutigkeit unberücksichtigt lassen, die sich darin zeigt, daß das für die Geltungssicherung zentrale Prädikat 'wahr' nicht nur (epistemologisch) als 'verifiziert' bzw. 'verifizierbar' gelesen werden kann, sondern (ontologisch) seinerseits wiederum auf eine semantische Basis - das Wahr-Sein für die pragmatische Verwendung - das Als-wahr-Erkennen - zu verweisen scheint. Diese Zweideutigkeit - sie läßt sich schon in antiken Texten, die sich mit Sprache beschäftigen, nachweisen (z.B. im Streit um den Status der Logik zwischen Stoa und Peripatos) - verdankt ihre moderne Wirksamkeit Frege, der 'wahr' sowohl semantisch wie pragmatisch benützt: semantisch nämlich ist Wahrheit eine der beiden möglichen Bedeutungen eines Gedankens, pragmatisch aber wird im Urteil, also in der Behauptung eines Gedankens, die Anerkennung seiner Wahrheit vollzogen. Bedeutet jedoch ein Gedanke das Falsche, so sieht man, daß der mit einer Behauptung erhobene Wahrheits a $\mathrm{n} \mathrm{p} \mathrm{ru} \mathrm{ch}$ nicht mit seiner Einlösung verwechselt werden darf. Freges Gleichbehandlung der assertorischen Sätze bzw. ihres Inhalts, d.i. ihres Gedankens, mit den Gegenstandsnamen läßt den von ihm selbst hervorgehobenen entscheidenden Unterschied unberücksichtigt, daß ein Gedanke noch zur Beurteilung ansteht, Namen hingegen nur eine semantische, keine unmittelbar pragmatische Rolle spielen. Von Wahrheit sowohl in semantischer wie in pragmatischer Hinsicht zu sprechen - Frege beruft sich auf die Notwendigkeit, die bloße Annahme der Wahrheit von ihrer Anerkennung unterscheiden zu müssen - mag der Grund dafür gewesen sein, daß der Ausdruck ' $A$ ' durchweg syntaktisch als Satzzeichen auftritt, obwohl )Einwortsätze ( naheliegende Beispiele dafür gewesen wären, an derselben Zeichengestalt einen Wortaspekt (=semantischen Aspekt) - Zeichen in ihrer repräsentativen Funktion und einen Satzaspekt (= pragmatischer Aspekt) - Zeichen in ihrer kommunikativen Funktion - zu unterscheiden. Und zu beachten ist, daß von einer Zeichengestalt als Wort ebensowenig sein Sinn abgelöst werden kann - es wäre kein Wort, kein verständliches Sprachzeichen mehr (obwohl ein als Wort vermutetes Lautschema auf seinen Sinn hin befragt werden kann) - wie von einer Zeichengestalt als Aussage ihre Geltung abgetrennt werden kann - sie wäre keine Aussage, kein verläßliches Sprachzeichen mehr (obwohl natürlich auch hier der bloße Geltungsanspruch auf seine Einlösbarkeit hin befragt werden kann). Wabr ist daher kein Prädikator zur Unterscheidung von Aussagen, ebensowenig wie sinnvoll ein Prädikator zur Unterscheidung von Prädikatoren ist, außer eben über bloßen Zeichengestalten, sie als Aussagen bzw. als Wort qualifizierend. Das aber war trotz der zusätzlichen verwirrenden Verwendung von 'wahr' (neben 'sinnvoll') im semantischen Zusammenhang bereits der Inhalt von Freges großartiger Einsicht in die Explizierbarkeit von Wahrheit allein im pragmatischen Zusammenhang. 
Es wäre nun vergeblich, durch eine Definition deutlicher zu machen, was unter 'wahr' zu verstehen sei. Wollte man etwa sagen: "Wahr ist eine Vorstellung, wenn sie mit der Wirklichkeit übereinstimmt", so wäre damit nichts gewonnen, denn, um dies anzuwenden, müßte man in einem gegebenen Falle entscheiden, ob eine Vorstellung mit der Wirklichkeit übereinstimme, mit andern Worten: ob es wahr sei, daß die Vorstellung mit der Wirklichkeit übereinstimme. Es müßte also das Definierte selbst vorausgesetzt werden. Dasselbe gälte von jeder Erklärung von dieser Form: "A ist wahr, wenn es die und die Eigenschaften hat, oder zu dem und dem in der und der Beziehung steht'. Immer käme es wieder im gegebenen Fall darauf an, ob es wahr sei, daß $A$ die und die Eigenschaften habe, zu dem und dem in der und der Beziehung stehe. Wahrheit ist offenbar etwas so Ursprüngliches und Einfaches, daß eine Zurückführung auf noch Einfacheres nicht möglich ist. Wir sind daher angeweisen, das Eigentümliche unseres Prädikats durch Vergleichung mit anderen ins Licht zu setzen. Zunächst unterscheidet es sich von allen anderen Prädikaten dadurch, daß es immer mit ausgesagt wird, wenn irgend etwas ausgesagt wird. Wenn ich behaupte, $\mathrm{da}$ die Summe von 2 und 35 ist, so behaupte ich damit, daß es wahr ist, daß 2 und 35 ist (...). Die Form des Behauptungssatzes ist also eigentlich das, womit wir die Wahrheit aussagen, und wir bedürfen dafür des Wortes 'wahr' nicht. Ja, wir können sagen: selbst da, wo wir die Ausdrucksweise "es ist wahr, daß ...." anwenden, ist eigentlich die Form des Behauptungssatzes das Wesentliche. (Logik, in: G. Frege, Nachgelassene Schriften, hrsg. H. Hermes u.a., Hamburg 1969, S. 139 ).

Kehren wir mit dieser Einsicht zum Unterschied partiebezogener von strategiebezogener Uberlegungen in der Dialoglogik zurück, so können wir diesen Unterschied terminologisch so auffassen, daß neben der Explikation der Argume ntat i on smögli chkeit e n umeine Aussage eine davon verschiedene Explikation der B e g r ü n d u n g s $\mathrm{mög} \mathbf{l}$ i chke it e n für eine Aussage a u $\mathrm{gru}$ nd der gegebenen Argumentationsmöglichkeiten erforderlich ist. Es wird dann deutlich, $\mathrm{da}$ die angemahnte Rechtfertigung für die Dialogspielregeln sowohl global - als Rahmenregel - wie lokal - als Argumenteregel, darunter insbes. die Partikelregeln - mit der Rechtfertigung für Begründungsmöglichkeiten, z.B. durch logisches oder auch inhaltliches Schließen, nämlich von Aussagen, den Hypothesen, auf eine andere Aussage, die These, nicht verwechselt werden darf.

An der Behandlung der Implikationen oder Wenn-dann-Aussagen, also der hypothetischen Urteile der Tradition, in Zeichen: A $<B$, läßt sich der Zusammenhang und der Unterschied von Argumentation und Begründung in ausgezeichneter Weise deutlich machen. Man muß dazu nur diejenigen Fälle, in denen Implikationen begründend verwendet werden, von denjenigen Fällen unterscheiden, in denen sie begründungsbedürftig bzw. begründungsherausfordernd verwendet werden, beide $\mathrm{Male}$ in $\mathrm{Zu}$ sammenhängen, wo über die Geltung des Implikans A nichts bekannt ist. 
Nehmen wir das Beispiel $\mathbf{C}$ ( $\rightleftharpoons$ Wenn N's Rasensprenger angestellt ist, dann ist $\mathrm{N}$ zuhause), so kann $\mathrm{C}$ begründend für ' $\mathrm{N}$ ist zuhause' eingesetzt sein, nämlich dadurch, daß etwas \ Sichtbares $〈$, das Rasensprengen, aufgrund einer notwendigen Verknüpfung, eben $\mathrm{C}$, als ) Zeichen ( für etwas JUnsichtbares 〈 verwendet wird (diese semiotische Terminologie geht auf die Logik der Stoa zurück), es kann aber $\mathrm{C}$ auch begründungsherausfordernd bzw. begründungsbedürftig eingesetzt sein und wird dann etwa durch die Aussage $\mathrm{D}(\rightleftharpoons$ Wenn $\mathrm{N}$ fortgeht, dann stellt $\mathrm{N}$ den Rasensprenger $\mathrm{ab}$ ) begründet, und zwar unter Benützung des dann seinerseits begründenden $\mathrm{D}<\mathrm{C}$, wobei $\mathrm{C}$ die Struktur $\mathrm{A} \rightarrow \mathrm{B}$ hat mit $\mathrm{A} \leftrightharpoons \mathrm{N}$ 's Rasensprenger ist angestellt und $\mathrm{B} \leftrightharpoons \mathrm{N}$ ist zuhause.

Im zweiten Fall ist $\mathrm{D}$, ebenso wie $\mathrm{C}$ im ersten Fall, unmittelbar durch einen Handlungszusammenhang bzw. das Wissen um ihn begründet, also praktisch und nicht mehr theoretisch. Man könnte dies durch eine pragmatische, also synthetische, Prädikatorenregel:

N's Fortgehen $<N$ 's Rasensprenger abstellen

ausdrücken, im Unterschied zu semantischen, also analytischen, Prädikatorenregeln, wie sie als terminologische Zusammenhänge oder Bedeutungspostulate in Wörterbüchern auftreten oder eigens vereinbart sein können, und die in besonderen, allgemein auf einen Sprachgebrauch in Alltag oder Wissenschaft bezogenen Fällen sich natürlich auch begründend heranziehen lassen.

Ein nur begründender Wenn-dann-Satz, der also nicht seinerseits begründungsherausfordernd oder begründungsbedürftig verwendet wird, muß nun seinerseits einen argumentativen Sinn bekommen, der im komplexen Fall nicht unmittelbar durch pragmatische oder gar semantische Prädikatorenregeln repräsentiert werden kann. Dieser argumentative Sinn eines begründend verwendeten Wenn-dann-Satzes soll durch eine $\mathrm{D}$ i a $\log$ b e di n g u ng für die Behandlung eines Satzes der Form $A \rightarrow$ B eingefangen werden:

Wer $A \rightarrow B$ behauptet, verpflichtet sichzurVerteidigung mit der Behauptung B a u f en Angriff $A$; diese ( $z$ unächst nur potentielle) Verteidigungspflichtsoll nichteher aktuell werden, als A seinerseits a u endlich viele Gegenangriffe verteidigt worden ist.

Wird diese Dialogbedingung akzeptiert, so ergeben sich zusammen mit den unproblematischen Dialogspielregeln für die übrigen logischen Partikeln diejenigen partiebezogenen Argumentationsregeln um Aussagen, die den argumentativen Sinn einer Aussage in der Dialoglogik ausmachen. 
Sie stellen daher diejenige pragmatische Basis bereit, auf die in strategiebezogenen Argumentationen, also Begründungen, mit dem Ziel, die Geltung einer Aussage zu sichern - sie wird dann nicht begründend sondern begründungsbedürftig bzw. begründungsherausfordernd verwendet zurückgegriffen werden muß. 